\title{
Improvement of the pion spectrum with HYP-smeared staggered fermions
}

\author{
Taegil Bae*, David Adams, Hyung-Jin Kim, Jongjeong Kim, Kwangwoo Kim, and \\ Weonjong Lee \\ Frontier Physics Research Division and Center for Theoretical Physics, \\ Department of Physics and Astronomy, Seoul National University, Seoul, 151-747, South Korea \\ E-mail: wlee@phya.snu.ac.kr
}

\section{Chulwoo Jung}

Physics Department, Brookhaven National Laboratory, Upton, NY11973, USA

E-mail: chulwoo@bnl.gov

\section{Stephen R. Sharpe}

Department of Physics, University of Washington, Seattle, WA 98195-1560, USA

E-mail: sharpe@phys.washington.edu

\begin{abstract}
We extend our previous study of taste-symmetry breaking using HYP-smeared staggered fermions in two ways. First, we improve the statistics of a comparison of unimproved and HYP-smeared staggered fermions on quenched lattices with $a \approx 0.1 \mathrm{fm}$. This allows us to obtain a signal for all pion tastes, rather than just a subset, and thus to make a complete comparison. In addition, it allows us to differentiate between wall and local sources. Second, we compare HYP-smeared valence quarks to asqtad valence quarks on $2+1$ flavor unquenched MILC lattices. We find that taste breaking is substantially reduced by HYP-smearing, bringing the size of this discretization effect (which is the dominant such effect with staggered fermions) down to the size expected generically for any fermion type.
\end{abstract}

The XXV International Symposium on Lattice Field Theory

July 30 - August 42007

Regensburg, Germany

${ }^{*}$ Speaker. 


\section{Introduction}

Unimproved staggered fermions suffer from very large discretization errors, particularly those that involve the breaking of taste symmetry. Reducing these errors is crucial for practical applications, and one popular choice is the asqtad action [1] which has been extensively used by the MILC collaboration. This is a tree-level $O\left(a^{2}\right)$ improved action, and has been found to significantly reduce taste breaking. Nevertheless, taste-breaking remains the dominant discretization error, and further improvement is desirable. This can be accomplished by the use of multiple levels of link-smearing (with reunitarization at each stage) [2,3]. A tree-level $O\left(a^{2}\right)$ improved action with multi-level smearing is the HISQ action [3] —and this is the present staggered action of choice for charm quarks. For light quarks, however, where $a^{2} m_{q}^{2}$ effects are likely small, we are pursuing a simpler option. This is the "HYP action", in which one uses HYP-smeared links (which have three levels of smearing) [2], but otherwise keeps the unimproved staggered action. This action is not fully $O\left(a^{2}\right)$ improved, but our hope is that it is effective at reducing the dominant taste-breaking discretization error down to the generic size, namely $a^{2} \Lambda_{\mathrm{QCD}}^{2}$.

Last year we presented our first results from a comparative study of taste-breaking with unimproved, HYP-smeared and asqtad valence fermions [4]. We used the splitting in the pion multiplet as a non-perturbative measure of taste breaking. We found that for quenched configurations with $a \approx 0.1 \mathrm{fm}$ there was a dramatic reduction in taste-breaking. Indeed, our results indicated that, rather than treating the splittings as a leading order effect in a joint chiral-continuum expansion, i.e. $a^{2} \sim p^{2}$, they should be treated as a next-to-leading-order effect, i.e. $a^{2} \sim p^{4}$. This would substantially simplify the formulae of staggered chiral perturbation theory $[5,6]$.

During the last year we have improved the statistics of the quenched calculation, allowing a more thorough calculation of the pion spectrum, now including all tastes, although the overall conclusions are unchanged. More importantly, we have calculated the pion spectrum with the valence HYP action on unquenched MILC "coarse" lattices $(a \approx 0.125 \mathrm{fm})$, allowing a direct comparison with results using valence asqtad action, and also allowing us to determine the power-counting appropriate for the HYP action.

We do not repeat here the technical discussion concerning the types of sources and sinks that we use, refering the interested reader to last year's talk [4]. We focus instead on the new numerical results and the corresponding conclusions. Further details will be given in an upcoming publication.

\section{Update on quenched results}

We use quenched lattices of size $16^{3} \times 64$ generated with the Wilson gauge action at coupling $\beta=6$, so that $1 / a \approx 1.95 \mathrm{GeV}$. Compared to last year, we have increased our statistics from 218 to 370 configurations. For unimproved fermions we use quark masses $a m_{q}=0.005,0.01,0.015$, $0.02,0.025$ and 0.03, while for HYP fermions we use $a m_{q}=0.01,0.02,0.03,0.04$, and 0.05 . Despite appearances, these ranges corresponds to heavier physical masses for unimproved than for HYP-smeared fermions. This is because $Z_{m} \approx 2.5$ for the former, while $Z_{m} \approx 1$ for the latter. For example, the physical strange quark mass is $a m_{s}^{\text {phys }} \approx 0.025$ for unimproved fermions and 0.052 with HYP smearing. 

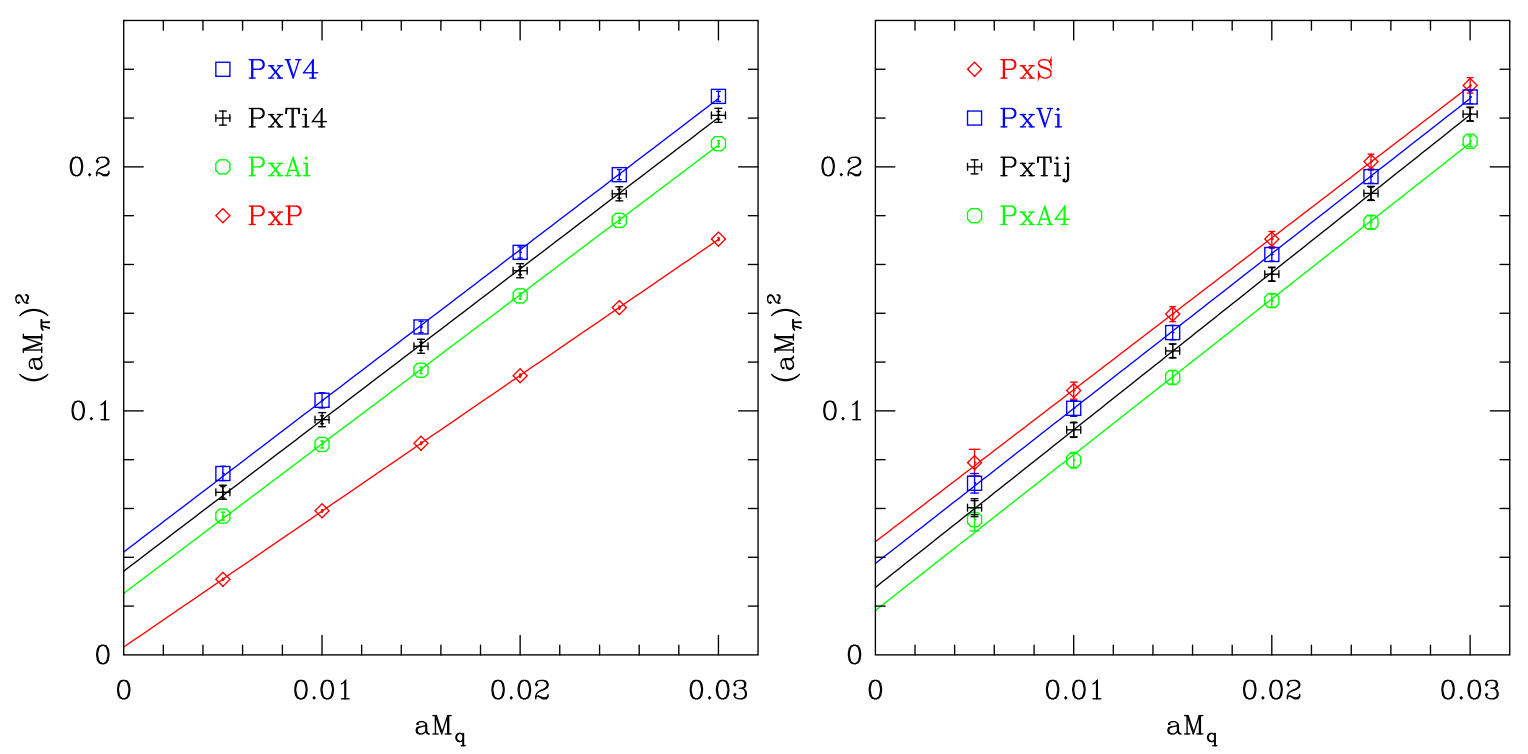

Figure 1: $\left(a m_{\pi}\right)^{2}$ vs. $a m_{q}$ for unimproved staggered fermions with cubic wall sources on quenched lattices. Left panel: LT tastes; Right panel: NLT tastes. Linear chiral extrapolations are shown.

The increase in statistics allows us to calculate the masses of pions of all tastes. Last year we had results only for the "LT" (local in time) tastes, i.e. $\xi_{5}, \xi_{4}, \xi_{i 5}$ and $\xi_{i 4}$. For pseudoscalar spin, $\gamma_{S}=\gamma_{5}$, these are the tastes created by an operator that is local in time. This year we have signals also for the "NLT" (non-local in time) tastes $\mathbf{1}, \xi_{i}, \xi_{45}$ and $\xi_{i j}$. These are produced by our sources (which reside on a single timeslice) by their coupling to the axial current, $\gamma_{S}=\gamma_{4} \gamma_{5}$, which is suppressed relative to the production of LT tastes.

As described last year, we use two choices of sink operators, and find them to be indistinguishable. All results presented here are obtained with the "Golterman" [7] sinks. We also use two sources - an extended "cubic wall source" and a local "cubic U(1) source". We find that both work comparably well for LT pions, while the cubic wall source leads to smaller errors for the NLT pions, although the improvement is smaller for the HYP action than for the unimproved action.

In Figs. 1 and 2 we show, respectively, the pion spectrum for unimproved and HYP-smeared staggered fermions. The dramatic reduction in the breaking of taste symmetry observed last year is seen to carry over to the NLT tastes. For all except the taste singlet, this result is expected because of the predicted approximate $\mathrm{SO}(4)$ symmetry, which holds at leading order in staggered chiral perturbation theory [5]. The $\mathrm{SO}(4)$ symmetry combines tastes $\xi_{i}$ (NLT) and $\xi_{4}$ (LT) into a single multiplet with taste $\xi_{\mu}$, and similarly for the $\mathrm{SO}(4)$ irreps with tastes $\xi_{5 \mu}$ and $\xi_{\mu \nu}$. Of the NLT tastes, only the taste singlet is unpaired, and thus the fact that its mass is very close to those of the other tastes does not follow from $\mathrm{SO}(4)$ symmetry, but rather is an additional, independent indication of the smallness of taste breaking with HYP fermions.

In Table 1, we list the mass-squared splittings after linear extrapolation to the chiral limit. These are proportional to $a^{2}$ in staggered chiral perturbation theory. Note that knowledge of $Z_{m}$ is not needed in order to compare these quantities between the two types of fermion. Our main conclusion is that taste breaking is reduced by an order of magnitude by HYP smearing. In fact, only for the LT tastes (the left columns) are the splittings significantly different from zero with 

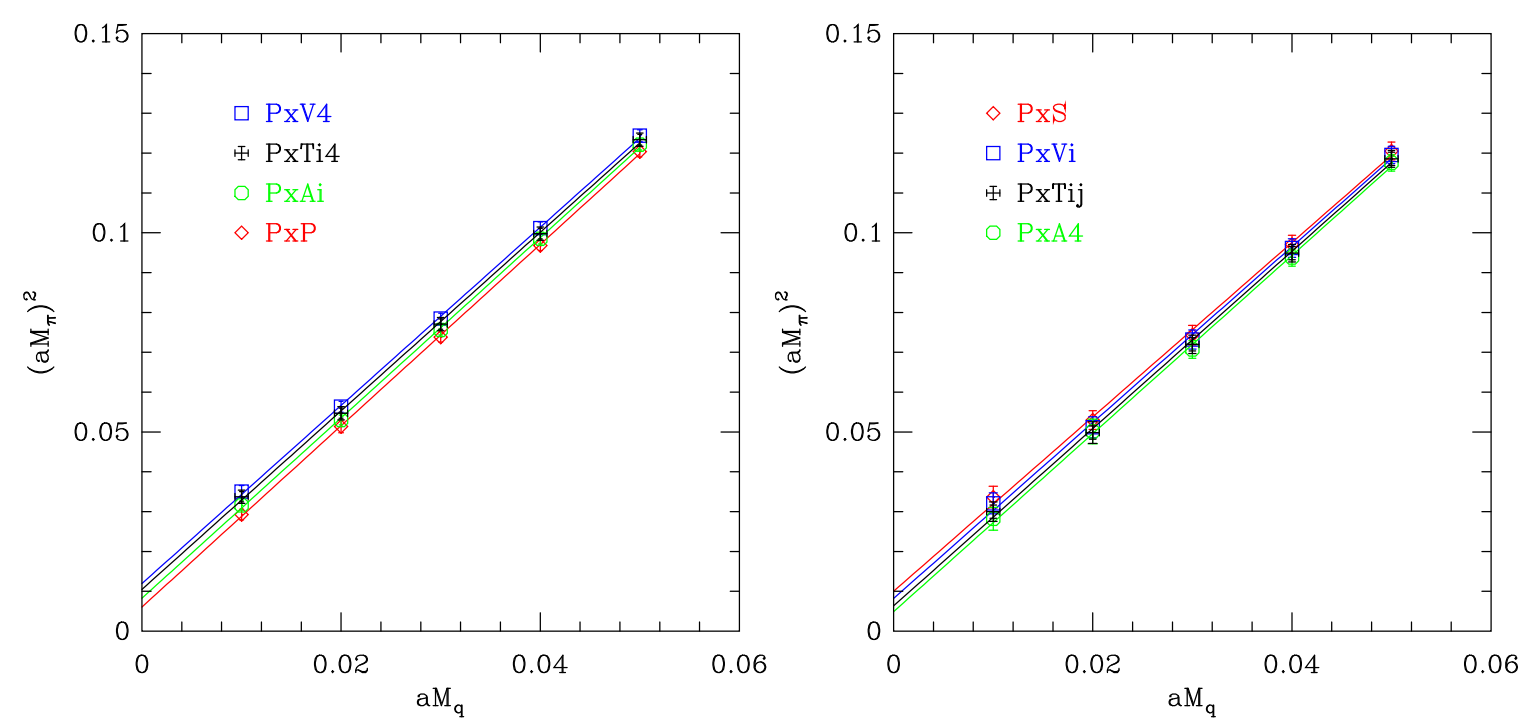

Figure 2: $\left(a m_{\pi}\right)^{2}$ vs. $a m_{q}$ for HYP-smeared staggered fermions with cubic wall sources on quenched lattices: Left panel: LT tastes; Right panel: NLT tastes. Linear chiral extrapolations are shown.

HYP smearing. For the NLT tastes, even the ordering of states is not clear. We also note that there is evidence for $\mathrm{SO}(4)$ symmetry breaking for unimproved fermions at the $2-3 \sigma$ level, with the NLT tastes systematically lower than their LT partners. We are investigating this issue further.

\begin{tabular}{ccc|ccc}
\hline Taste $(F)$ & $\Delta_{F}$ (unimproved) & $\Delta_{F}$ (HYP) & Taste $(F)$ & $\Delta_{F}$ (unimproved) & $\Delta_{F}(\mathrm{HYP})$ \\
\hline$\xi_{i 5}$ & $0.0230(09)$ & $0.0022(09)$ & $\xi_{45}$ & $0.0200(34)$ & $-0.0012(29)$ \\
$\xi_{i 4}$ & $0.0360(24)$ & $0.0044(10)$ & $\xi_{i j}$ & $0.0245(40)$ & $0.0003(31)$ \\
$\xi_{4}$ & $0.0447(27)$ & $0.0059(22)$ & $\xi_{i}$ & $0.0342(42)$ & $0.0022(30)$ \\
& & & $I$ & $0.0432(44)$ & $0.0040(30)$ \\
\hline
\end{tabular}

Table 1: Comparison of taste symmetry breaking with unimproved and HYP-smeared fermions on quenched lattices with cubic wall sources. Here $\Delta_{F}=\left[a_{\pi}(F)\right]^{2}-\left[a m_{\pi}\left(\xi_{5}\right)\right]^{2}$, extrapolated to the chiral limit.

In conclusion, we confirm and extend the findings of last year. Unimproved staggered fermions show taste breaking that is of the same order as the light pion masses, so that $\mathscr{O}\left(a^{2}\right) \sim \mathscr{O}\left(p^{2}\right)$ is the appropriate power counting. Furthermore, the slopes differ significantly for the different tastes, indicating an important next-to-leading-order (NLO) contribution from $\mathscr{O}\left(a^{2} p^{2}\right)$ terms. By contrast, for HYP-smeared fermions both the splittings and the differences in the slopes are much smaller. Thus we conclude that one should treat $\mathscr{O}\left(a^{2}\right)$ effects as being of NLO for HYP fermions on these lattices. The important practical question is whether this holds also for unquenched lattices.

\section{Comparison of asqtad and HYP-smeared staggered fermions}

To address this we have compared asqtad and HYP-smeared staggered valence fermions on one set of the coarse $2+1$ flavor MILC lattices [8]. The parameters of the study are given in Table 2. The range of quark masses for asqtad quarks is similar to that used above, the physical 


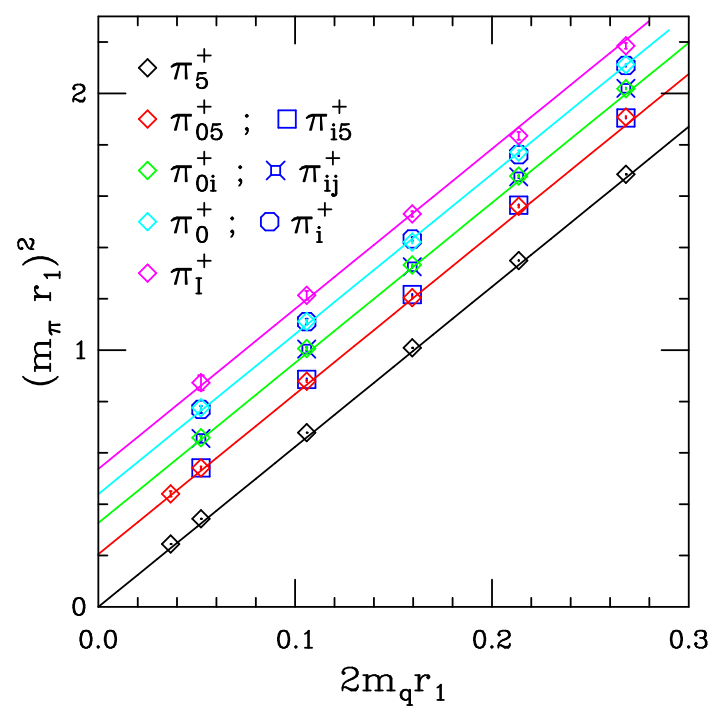

Figure 3: $\left(r_{1} m_{\pi}\right)^{2}$ vs. $2 r_{1} m_{q}$ for asqtad valence quarks on unquenched lattices. From Ref. [9].

strange quark mass $a m_{s}^{\text {phys }} \approx 0.045$ lying near the upper end. It turns out, however, that the HYP quarks we use are somewhat lighter. For the same bare quark mass, the physical quark mass is about 1.4 times smaller with HYP than with asqtad quarks. Part of this difference is due to the normalization convention for tadpole improvement used by Ref. [9], which has the effect that the bare quark mass for asqtad quarks should be multiplied by $1 / u_{0}$ to match the convention used for unimproved and HYP quarks. Here $u_{0}$ is the "mean link", which is $\approx 0.87$ for these lattices. The remainder of the difference is presumably due to a difference in the values of $Z_{m}$ for the two lattices.

In Fig. 3, we show the results obtained in Ref. [9] using asqtad valence quarks. One sees that taste-breaking is significant, with $\mathscr{O}\left(a^{2}\right) \sim \mathscr{O}\left(p^{2}\right)$ being the appropriate power counting. Note that the taste-breaking with unimproved staggered quarks would be larger still, since these lattices are coarser than the quenched lattices used earlier. Despite the relatively large taste breaking,

\begin{tabular}{cc}
\hline parameter & value \\
\hline gauge action & 1-loop tadpole-improved Symanzik \\
sea quarks & $2+1$ flavors of asqtad staggered $\left(a m_{\ell}=0.01, a m_{s}=0.05\right)$ \\
$\beta$ & 6.76 \\
$a$ & $0.125 \mathrm{fm}$ \\
geometry & $20^{3} \times 64$ \\
$\#$ of confs & $640($ asqtad $) / 406(\mathrm{HYP})$ \\
valence quark type & asqtad and HYP staggered \\
valence quark mass (asqtad) & $0.007,0.01,0.02,0.03,0.04,0.05$ \\
valence quark mass (HYP) & $0.05,0.01,0.015,0.02,0.025,0.03,0.035,0.04,0.045,0.05$ \\
\hline
\end{tabular}

Table 2: Simulation parameters used for the comparison of asqtad and HYP valence quarks. Asqtad results are from Ref. [9]. 

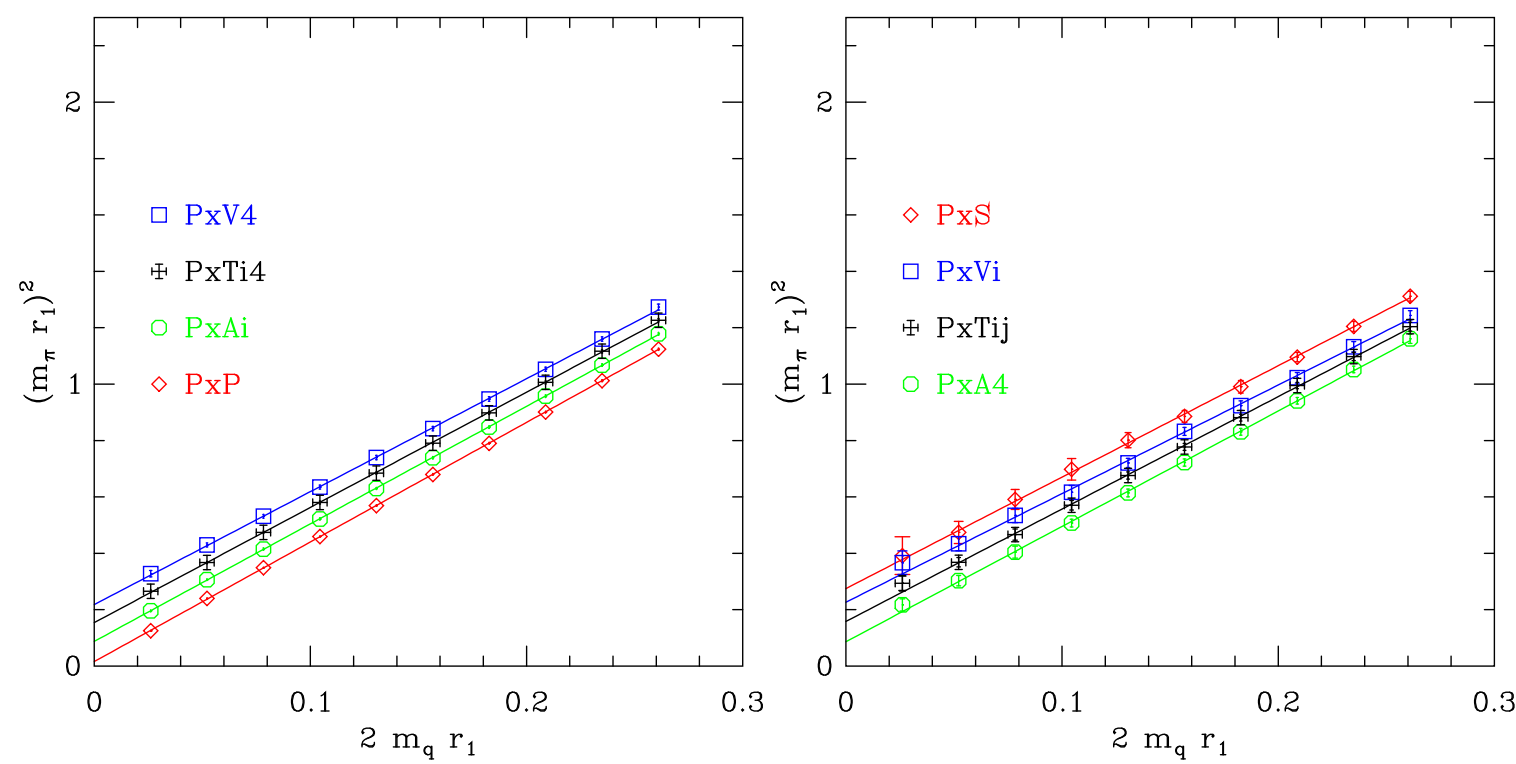

Figure 4: $\left(r_{1} m_{\pi}\right)^{2}$ vs. $2 r_{1} m_{q}$ for HYP valence quarks on unquenched lattices, using cubic U(1) sources. Left panel: LT tastes; Right panel: NLT tastes.

discretization effects of NLO (i.e. $\mathscr{O}\left(a^{2} p^{2}\right)$ ) are very small. This is shown both by the lack of breaking of $\mathrm{SO}(4)$ symmetry, and the result that the slopes for different tastes are close. The latter result differs from that found above with unimproved fermions.

Our new results using HYP-smeared staggered quarks are shown in Fig. 4. Results are with cubic U(1) sources; those with cubic wall sources are not yet available. We plot using the same dimensionless variables as in Fig. 3. The difference in slopes between asqtad and HYP fermions (by a factor of about 1.4) is due to the difference in quark mass conventions and renormalization factors discussed above. Irrespective of this detail, we see that taste breaking is substantially reduced for HYP-smeared quarks. To make this quantitative we quote, in Table 3, the splittings in the chiral limit (using $r_{1}$ to set the scale, rather than the $1 / a$ used above). We see that HYP smearing reduces taste breaking by a factor of 2.5-3, although the ordering of states is the same for both asqtad and HYP actions. The improvement in taste-breaking is similar to that found using the HISQ action [3].

\begin{tabular}{ccc|ccc}
\hline Taste $(F)$ & $\Delta_{F}($ asqtad $)$ & $\Delta_{F}(\mathrm{HYP})$ & Taste $(F)$ & $\Delta_{F}($ asqtad $)$ & $\Delta_{F}(\mathrm{HYP})$ \\
\hline$\xi_{i 5}$ & $0.205(2)$ & $0.072(2)$ & $\xi_{45}$ & $0.205(2)$ & $0.070(18)$ \\
$\xi_{i 4}$ & $0.327(4)$ & $0.138(4)$ & $\xi_{i j}$ & $0.327(4)$ & $0.142(20)$ \\
$\xi_{4}$ & $0.439(5)$ & $0.203(7)$ & $\xi_{i}$ & $0.439(5)$ & $0.210(28)$ \\
& & & $I$ & $0.537(15)$ & $0.259(39)$ \\
\hline
\end{tabular}

Table 3: Taste symmetry breaking effect: $\Delta_{F}=\left[r_{1} m_{\pi}(F)\right]^{2}-\left[r_{1} m_{\pi}\left(\xi_{5}\right)\right]^{2}$ extrapolated to the chiral limit. Results from unquenched lattices comparing valence asqtad (from Ref. [9]—with $\mathrm{SO}(4)$ symmetry enforced) to HYP-smeared fermions (with cubic U(1) sources).

We can characterize the taste-breaking errors in terms of a non-perturbative scale. Using the splitting of the "2-link" pions (taste $\xi_{\mu v}$ ) from the Goldstone pion as an "average" splitting, 
and setting $\Delta a^{2} m_{\pi}^{2}=a^{4} \Lambda^{4}$, we find $\Lambda \approx 0.6 \mathrm{GeV}$. This should be compared to scales $\approx 1 \mathrm{GeV}$ for unimproved staggered fermions. Thus improvement has reduced discretization errors to the expected generic size, i.e. $\Lambda \sim \Lambda_{\mathrm{QCD}}$.

Looking in more detail at the results for HYP fermions, we note that $\mathrm{SO}(4)$ breaking is small, and that the slopes of $m_{\pi}^{2}$ versus $m$ graphs are similar for all tastes. Thus, as for the asqtad quarks, the NLO $\mathscr{O}\left(a^{2} p^{2}\right)$ terms responsible for these effects must be small.

Despite the reduction in taste breaking, it is clear from the figures that, for this range of quark masses (which is the range we are using for calculating matrix elements), the appropriate power counting for the MILC coarse lattices remains $\mathscr{O}\left(a^{2}\right) \sim \mathscr{O}\left(p^{2}\right)$ for HYP fermions.

\section{Conclusions}

The most important conclusion for future work is that HYP smearing leads to a significant reduction in taste breaking compared to the asqtad action. This was expected from previous studies, but it is important to demonstrate this on the unquenched MILC lattices that we are using for calculating weak matrix elements. The reduction is, however, not small enough to change the power counting for staggered chiral perturbation theory on the coarse lattices. If, however, one extrapolates to the fine MILC lattices, on which one expects that taste breaking will be reduced by about 2.5 (which is the relative size of $\alpha^{2} a^{2}$ ), then it seems likely that one will be able to treat the discretization errors as a NLO effect, i.e. that $\mathscr{O}\left(a^{2}\right) \sim \mathscr{O}\left(p^{4}\right)$. Pictorially, the spectrum on the fine lattices should look like that for the quenched lattice results of Fig. 2. This would lead to a significant simplification in the fitting forms from staggered chiral perturbation theory.

\section{Acknowledgment}

W. Lee acknowledges with gratitude that the research at Seoul National University is supported by the KICOS grant K20711000014-07A0100-01410, by the KRF grant KRF-2006-312-C00497, by the BK21 program, and by the US DOE SciDAC-2 program. The work of S. Sharpe is supported in part by the US DOE grant no. DE-FG02-96ER40956, and by the US DOE SciDAC-2 program.

\section{References}

[1] K. Orginos et al., Phys. Rev. D60 (1999) 054503, [hep-lat/9903032]; G.P. Lepage, Phys. Rev. D59 (1999) 074502, [hep-lat/9809157].

[2] A. Hasenfratz and F. Knechtli, Phys. Rev. D64 (2002) 034504, [hep-lat/0103029].

[3] E. Follana et al. [HPQCD Collaboration], Phys. Rev. D 75, 054502 (2007) [arXiv:hep-lat/0610092].

[4] Taegil Bae et al., PoS LAT2006 (2006) 086, [hep-lat/0610057].

[5] Weonjong Lee and Stephen Sharpe, Phys. Rev. D60 (1999) 094503, [hep-lat/9905023].

[6] C. Aubin and C. Bernard, Phys. Rev. D68 (2003) 034014, [hep-lat/0304014].

[7] Maarten Golterman, Nucl. Phys. B273 (1986) 663-676.

[8] C. Bernard et al., Phys. Rev. D64 (2001) 054506, [hep-lat/0104002].

[9] C. Aubin et al., Phys. Rev. D70 (2004) 114501, [hep-lat/0407028]. 